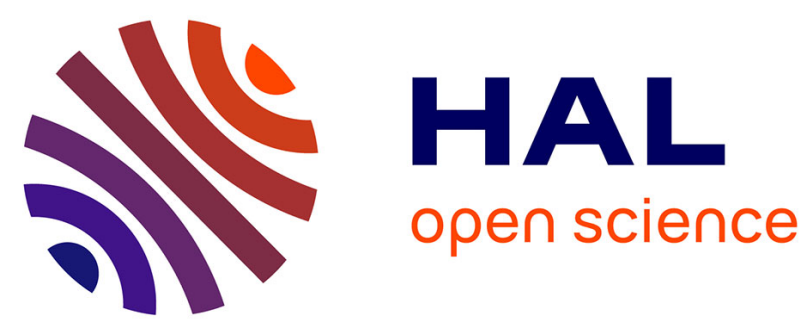

\title{
Examining the Effects of Height, Velocity and Emotional Representation of a Social Transport Robot and Human Factors in Human-Robot Collaboration
}

Jana Jost, Thomas Kirks, Stuart Chapman, Gerhard Rinkenauer

\section{- To cite this version:}

Jana Jost, Thomas Kirks, Stuart Chapman, Gerhard Rinkenauer. Examining the Effects of Height, Velocity and Emotional Representation of a Social Transport Robot and Human Factors in HumanRobot Collaboration. 17th IFIP Conference on Human-Computer Interaction (INTERACT), Sep 2019, Paphos, Cyprus. pp.517-526, 10.1007/978-3-030-29384-0_31. hal-02544598

\author{
HAL Id: hal-02544598 \\ https://hal.inria.fr/hal-02544598
}

Submitted on 16 Apr 2020

HAL is a multi-disciplinary open access archive for the deposit and dissemination of scientific research documents, whether they are published or not. The documents may come from teaching and research institutions in France or abroad, or from public or private research centers.
L'archive ouverte pluridisciplinaire HAL, est destinée au dépôt et à la diffusion de documents scientifiques de niveau recherche, publiés ou non, émanant des établissements d'enseignement et de recherche français ou étrangers, des laboratoires publics ou privés.

\section{(ㄷ)(i)}

Distributed under a Creative Commons Attribution| 4.0 International License 


\title{
Examining the Effects of Height, Velocity and Emotional Representation of a Social Transport Robot and Human Factors in Human-Robot Collaboration
}

\author{
Jana Jost ${ }^{1}$, Thomas Kirks ${ }^{1}$, Stuart Chapman ${ }^{1}$, and Gerhard Rinkenauer ${ }^{2}$ \\ 1 Fraunhofer Institute for Material Flow and Logistics, \\ Joseph-von-Fraunhofer-Str. 2-4, 44227 Dortmund, Germany \\ firstname. lastname@iml. fraunhofer.de \\ 2 Leibniz Research Centre for Working Environment and Human Factors, \\ Ardeystraße 67, 44139 Dortmund, Germany \\ lastname@ifado.de
}

\begin{abstract}
In nowadays industrial facilities robots play a major part in assisting the human worker. To ensure an effective process not only safety aspects have to be considered. For increasing the acceptance of humans towards robots, social aspects are important as well. In this paper we examine how the behaviour of humans towards other humans can be adapted to the robots behaviour. Especially, the usage of social rules regarding the distance between people are studied with different robot characteristics for height, velocity and emotional expressions as well as human factors.
\end{abstract}

Keywords: Empirical studies in HCI · User Experience Design · Social Robots

\section{Introduction}

Human-robot collaboration (HRC), a field of human-robot interaction (HRI), was established in various work fields. Most robots installed in warehouses are doing monotonous work and are surrounded by fences to ensure the safety of workers. Further, these robots do not mirror human behaviour and therefore cannot correctly be interpreted by the human which leads to discomfort [7]. However, to foster HRC in this field of application, the robot has to move autonomously and react according to its environment including the human. An essential factor for an effective collaboration is that the human feels safe, comfortable and interprets the actions of the robot correctly. A straightforward assumption is that people will approach social robots the same way as they would do with other people, thereby extending the computers are social actors theory [9] [19] to HRI. In approaching its user, the social theory of proxemics [9] has to be maintained to this goal. Here, two classifications of "safety" will be scrutinized. The physical safety focuses on avoiding collisions and is a decisive requirement 
for autonomous robots in HRC. The mental safety means ensuring that the robot does not cause fear for people. Mental safety is as important as physical safety [16]. If the presence or actions of the robot cause fear, the human will feel uncomfortable and use avoidance techniques to compensate the behaviour of the robot, although the physical safety is secured. For a pleasant and effective interaction, it is crucial that the robots meet the needs and expectations of the user. In particular, the robot needs to match social norms. In this case the proxemics is examined which can help to ensure mental safety. The examined social robot has the ability to show different faces via a display according to it's state, which plays a major role in human communication [5]. The impact of facial expressions in human and robot interaction has to be analysed. Emotional representations in robotics may add a new element in HRC and can strengthen the acceptance of robots [6]. By giving information about the robot's emotional state, the user will be able to predict the robots behaviour and therefore feel comfortable. The findings may contribute to a change in implementation of social robots in order to realise a better individualisation of robots when interacting with humans in a more personal manner.

\section{Background}

\subsection{Human-Robot-Interaction}

Today, robots can be found in various fields of application. Their tasks and capabilities have changed in the last decades from heavy industrial robots to more flexible and cognitive robots[22]. These offer direct interaction with the human without safety equipment and therefore are used also in other applications e.g. surgery [18]. Although the usage of robots in industrial applications is increasing, the human worker will always play a key role [13]. Therefore, the development of new interaction modalities for humans and robots is important.

According to ISO 8373 [12] HRC is defined as the exchange of information or actions between humans and robots to fulfil a given task. It is one interaction form besides cooperation and co-existence in HRI. In HRC humans and robots have the same goal or subgoals, for what coordination methods are needed. [18] For designing robots for HRC one has to consider various guidelines e.g. DIN EN ISO 10218 [8]. Those guidelines focus on safety aspects. Robots available on the market for HRC like ABB Dual-Arm [15] already fulfil the safety criteria but for HRC further issues e.g. psychological ergonomics have to be regarded to maximize the acceptance of humans towards robots.

\subsection{Proxemics}

As autonomous robots are collaborating with its human colleague, it is important to ensure safety and acceptance of the user. Hall [9] introduced the theory of proxemics and stated that people have distinct spaces surrounding them in elliptic circles. These four spaces are called intimate $(0.15 \mathrm{~m}-0.45 \mathrm{~m})$, personal 
$(0.45 \mathrm{~m}-1.2 \mathrm{~m})$, social $(1.2 \mathrm{~m}-3.6 \mathrm{~m})$ and public $(>3.6 \mathrm{~m})$. Each is defined by the kind of people entering the area. For example, partners and good friends are in the intimate or personal zone, whereas in the social zone acquaintances are positioned. A problem occurs as one enters a space he is not allowed to. The person feels anxious and might use avoidance techniques e.g. distancing himself from the other person. This avoidance behaviour is stated in the intimacy equilibrium model by Argyle [2]. Therefore, the robot needs to detect these zones and react in a manner that the human feels safe and comfortable.

An experiment examining the effects of three robot sizes on anxiety, conducted by Hirori and Ito [10], showed that participants felt more anxious and kept the greatest distance with the tallest robot $(1.8 \mathrm{~m})$. Concerning the robot velocity, Sakai et al. [20] revealed that the subjective distance kept to the robot correlated proportionally with the moving speed of the robot. They analysed five velocities ranging from $0.2 \mathrm{~m} / \mathrm{s}$ to $1.0 \mathrm{~m} / \mathrm{s}$. Velocities equal and greater than $0.8 \mathrm{~m} / \mathrm{s}$ caused anxiety. Ikeura et al. [11] confirmed this by analysing galvanic skin response and evaluating subjective ratings. The main aim of proxemics in HRI focuses on maximizing the user experience and enhancing the productivity of the collaboration. Human factors that determine how people react to approaching robots are their general attitudes towards robots [21], their experience in robotics, gender and age [7]. Other factors that have an impact on the distance between the two agents are the height, the velocity and mutual gaze of the robot.

\subsection{Analysis Methods}

In the following section the questionnaires will be outlined and general information will be given about these. The User Experience Questionnaire (UEQ), developed by Laugwitz et al. [14], was designed to enable a rapid assessment by the user, based on a comprehensive impression of the user experience. It should enable users to express feelings that arise during the experience with the product in a simple way. The UEQ is a valid method to evaluate how a robot is being perceived by the participants.

Normura and Kanda [17] proposed a concept to measure anxiety towards robots, resulting in the negative attitude towards robots scale (NARS). This scale was successfully used to explain differences in the behaviour of the participants in HRI studies. The Godspeed questionnaire was developed by Bartneck [3] as an HRI-specific measure of participants' perception across multiple dimensions, each one being applied with a set of semantic differential scales. We used the Godspeed questionnaire to measure the participants' perception of the robot.

\section{Research Hypotheses}

The intention of the socio-technical system, consisting of a social, autonomous transport vehicle (ATV) and the human worker is to provide an agreeable working environment, where the worker can fulfil his tasks and is supported by the 
ATV. According to the proxemic concept of Hall [9], we expect that the distance control of humans to a robot is influenced by factors of the robot, which are characterized either by the threat of the own safety or by social aspects. Aspects of safety, for example, refer to the size and speed of the robot, whereas social aspects are influenced by emotional expression. For this reason, we hypothesize the following points:

1. The speed of the robot affects the personal space. Faster robots lead to a greater distance between user and robot than slower ones.

2. The height of the robot affects the personal space. Smaller robots can enter further into the personal space than higher robots.

3. The facial expression of the robot influences the distance kept. A "happy" robot can enter further into the personal space than a sad one (Fig. 1).

4. The last point investigates the difference between human-robot and robothuman approach. The distance kept is smaller if the participant approaches than if the robot does.

\section{Methodology}

A laboratory experiment was conducted to explore what effects the height, the velocity and the two emotions happiness and sadness have on the distance between a social ATV and the participant.

\subsection{Experimental Setup}

In this study, the interaction with the social ATV is in the scope of research. It has the size of a small load carrier and is able to drive autonomously. The social ATV is designed for collaborating with human workers. With the integrated scissor lift, the carried load can be extended up to an ergonomic height.

Through webservices, the user can acquire its state or control it via wearables. The ATV has an e-paper display at its front. It displays emoji like faces, pictograms and textual hints to express the state of the robot in a comprehensible way. We are interested if humans notice the change of the emotional expression on its display and further if a significant difference is measurable between the two states, "happy" and "sad" (see Fig. 1a) and b)). While the robot expresses emotions in a human-like manner, we are interested if a human-like relationship is being created and social human-human norms are being transferred onto the robot. Further, we investigate the dichotomy between utility and pleasure in the ATV's height. According to ergonomic reasons, the robot can heighten its lift from $220 \mathrm{~mm}$ to $820 \mathrm{~mm}$, also while driving. Now the question arises, if the user is pleased or feels uncomfortable and distances himself from the robot or approaches the robot from the side, which may cause longer working operations and decreases usability.

We prepared an area of four by four meters and installed the OptiTrack [1] motion tracking system with multiple cameras to track the ATV and the head 


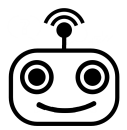

(a)

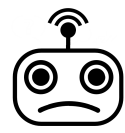

(b)

Fig. 1: Social ATV's face is either a) happy or b) sad

of the participant. Therefore, we attached markers on the ATV's front and the participant is wearing a marker beanie. Hence, with the recorded data from the tracking system the distances between ATV and human can be calculated. Inside the tracking area we installed an optical guidance track on the ground which the ATV uses to follow a straight track to approach the human at different velocities.

\subsection{Experimental Procedure}

Each participant received an introduction to the anonymised experiment. They could stop the experiment at any time. Before the experiment started, the participants provided their demographic variables. Afterwards, the participants were asked to stand on a specific position in the laboratory setting, which provided a distance of three meters to the ATV. The factors height, velocity and emotion are varied to mitigate an order bias. For example, the ATV is driving with a velocity of $0.4 \mathrm{~m} / \mathrm{s}$, an extended lift and represents a happy emotional state. The tasks involve approaching the ATV or being approached in the altered conditions. With a push button, the participant can stop the ATV from approaching in case of discomfort. Fig. 2 illustrates the testing environment. During the experiment, the movement of the social ATV as well as of the participant were recorded by using OptiTrack. Since, the ATV is using a new approach of interaction, participants could have a negative tendency towards the technology itself and therefore negative emotions could emerge towards the ATV. Hence, after the experiment, the participant filled in the UEQ "Attractiveness" Scale, NARS and the Godspeed questionnaire.

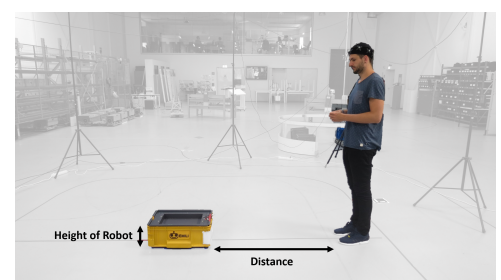

(a)

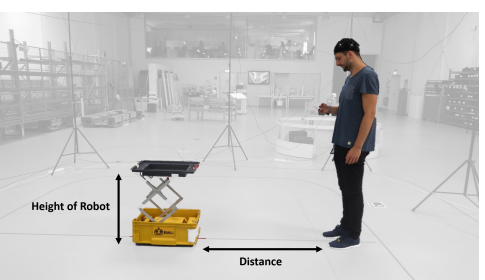

(b)

Fig. 2: ATV (left) and test subject (right). a) retracted lift b) extended lift 


\section{Results}

Sixteen participants took part in the experiment ( 3 females, 6 with experience in robotics) and the mean age was $31(\mathrm{SD}=9.04)$ years. We analyzed the two conditions separately (robot approaches participant vs. participant approaches robot).

\section{Robot approaches participant}

An ANOVA with the within subject factors velocity $(0.4 \mathrm{~m} / \mathrm{s}$ vs. $0.6 \mathrm{~m} / \mathrm{s})$, height (low vs. high) and emotion (happy vs. sad) and the between subject factor robot experience (no, yes) was conducted. In agreement with our hypothesis, the distance was closer when the robot was low $(449 \mathrm{~mm})$ than when the robot was high $(504 \mathrm{~mm}), \mathrm{F}(1,14)=6.13, \mathrm{p}=.03$. A significant interaction between robot experience and emotion, $\mathrm{F}(1,14)=8.3, \mathrm{p}=0.01$, suggests that the influence of the robot's emotional expression is opposite for the two robot experience groups (cf. Fig. 3a)). Separate ANOVAs for each factor levels of robot experience, however, reveal that the emotion expression of the robot influenced the distance for the participants with no prior robot experience, $\mathrm{F}(1,9)=5.9$, p $=.04$, but showed only a trend for the participants with robot experience, $\mathrm{F}(1,5)=4.6$, $\mathrm{p}=.08$. In line with our hypotheses the inexperienced group tolerates a shorter distance of the robot with a happy face. Interestingly, in the experienced group the influence of emotional expression seems to be damped or even inverted. Such a result can also mean, however, that for experienced participants the expressiveness of the facial features was not strong enough. With regard to the speed of the robot, our first hypothesis could not be confirmed, since the speed manipulation is not reflected in the behaviour of the test persons.

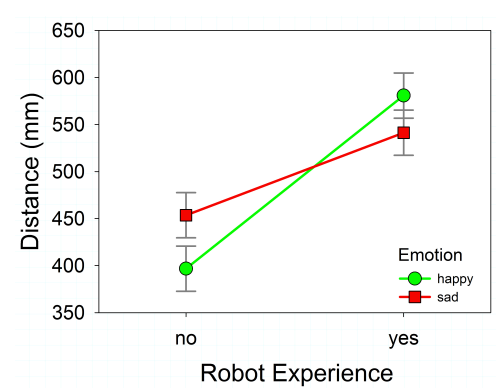

(a)

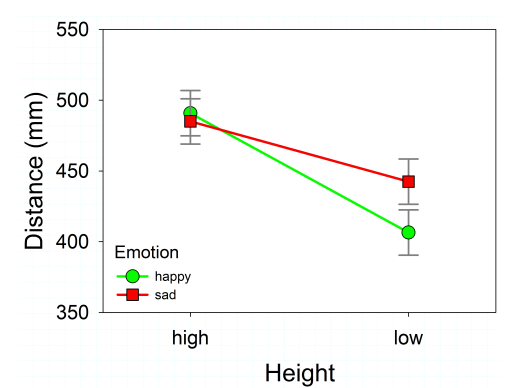

(b)

Fig. 3: Distance as a function of a) Robot Experience and Emotion and b) Height and Emotion. The error bars represent Fisher's least significant difference (LSD)

\section{Participant approaches robot}

ANOVAs for the within subject factors height and emotion and the between subject factor robot experience were conducted. Because the approaching velocity of the participants was not manipulated, the factor velocity is not relevant. To compare the situations in which either the participants move towards the 
robot or the robot moves towards the participants, the within factor movement condition (human vs. robot) was defined. Since the participants approached the robot only slowly, the slow robot movement condition $(0.4 \mathrm{~m} / \mathrm{s})$ was chosen as comparison. Again a significant main effect of factor height is revealed in the expected direction, $\mathrm{F}(1,14)=9.7, \mathrm{p}<.01$ (cf. Fig. $3 \mathrm{~b})$ ). The interaction between emotion and height just failed to be significant, $\mathrm{F}(1,14)=4.7, \mathrm{p}=.057$. Fig. $3 \mathrm{~b}$ ) depicts this interaction, whereby the mean values suggest that the emotional expression of the robot only affects the distance of the low robot, viz. the distance to the happy robot was shorter.

Finally, the analysis of variance revealed a three-way interaction, $\mathrm{F}(1,14)=$ $4.7, \mathrm{p}=.049$, which is depicted in Fig. 4 . This results suggest different patterns for the two robot experience groups. Separate ANOVAs for the two groups revealed no significant effects of factor height and movement condition for the experienced group, p's $>.1$, which may also be due to the low statistical power because of the small group size. For the inexperienced group there is a main effect for the factor height, $\mathrm{F}(1,9)=7.8, \mathrm{p}=.02$, viz. for the low robot a closer distance is tolerated. Furthermore, there was a trend for the interaction of movement condition and height, $\mathrm{F}(1,9)=3.4, \mathrm{p}=.097$, which may suggest that the distance control for the low and high robot size differs when the participants move towards the robot in comparison when the robot approaches the participants. In sum, the analyses of the moving participants in comparison to the moving robot provide additional insights into the HRI. The results suggest that the influence of the emotional expression of the robot on the proximity behaviour of the participants depends on the height of the robot. Furthermore, people who have no prior experience with robots seem to be more sensitive in regulating their distance as a function of robot height when moving towards the robot than people who have already prior experience.

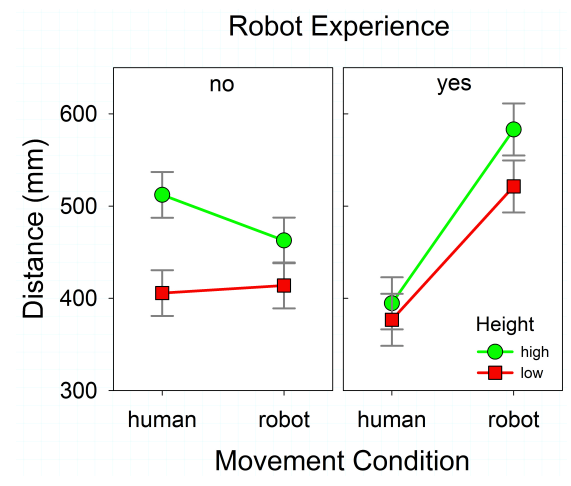

Fig. 4: Distance as a function of Movement Condition, Height and Robot Experience. The error bars represent Fisher's LSD.

The results of the "Attractiveness Scale" of the UEQ revealed a positive rating of the robot (Mean $=0.83, \mathrm{SD}=0.64, \mathrm{CI}=[0.48,1,18])$. Overall, the robot was rated as sympathetic and friendly. Analysis of NARS "Negative Attitudes 
toward Situations and Interactions with Robots" suggests rather positive attitudes in our experimental situation towards the robot, Mean $=3.7, \mathrm{SD}=0.8$, $\mathrm{CI}=[3.3,4.1]$. The analysis of "Negative Attitude toward Social Influence of Robots" revealed a rather neutral attitude of the participants with regard to the social influence, Mean $=3.0, \mathrm{SD}=0.7, \mathrm{CI}=[2.7,3.4]$. The reversed "Negative Attitudes toward Emotions in Interaction with Robots" suggests also a rather neutral attitude towards the robot used in this study, $\mathrm{M}=3.0, \mathrm{SD}=0.7$, CI $=[2.6,3.4]$. The Godspeed survey is divided into four factors: perceived safety $($ Mean $=3.5, \mathrm{SD}=0.5, \mathrm{CI}=[3.3,3.8])$, likeability $($ Mean $=4.0, \mathrm{SD}=0.6, \mathrm{CI}$ $=[3.7,4.3])$, anthropomorphism $($ Mean $=2.5, \mathrm{SD}=0.8, \mathrm{CI}=[2.0,2.9])$ and animacy $($ Mean $=2.8, \mathrm{SD}=0.5, \mathrm{CI}=[2.5,3.1])$. In sum, the subjective ratings suggest that the robot rated rather sympathetic and pleasant. The perceived safety was slightly positive and the likeability scale supports the findings from the UEQ that the robot was perceived as a rather convenient interaction partner but, however, not as a human counterpart.

\section{Discussion}

The factors are discussed in an order according to the importance of the findings. As stated in other studies, several factors of the robot and the personal background of the human have impact on the distance kept. The main factor in our study influencing the distance has been the height of the robot. Participants kept smaller distances to the robot with retracted lift $(448 \mathrm{~mm})$ than with extended lift $(503 \mathrm{~mm})$. Therefore, the second hypothesis can be confirmed whereas the first hypothesis cannot be verified. One reason could be that the given velocities were not fast enough (see findings [20]). Our fourth assumption refers to the approaches, robot approaching human vs. human approaching robot. As a result, people kept smaller distances, when they approached the ATV. According to prior robotic experience, the analysis revealed that participants kept greater distance to the robot. Without robotic experience, the facial representation of the social ATV played a role. They were excited to collaborate with a robot whereas people with robotic experience tended to show less excitement and might payed no attention to the display. This is only valid as the ATV does not extend its lift. When extended, the facial expressions had no influence, because people focused on the highest point of the ATV and ignored the display. Therefore, the third hypothesis can just partially be validated. According to the social theory of proxemics, the robot entered the personal space, in some cases even the intimate space. The intimate zone is supposed for familiar people. This may be caused by the appearance of the ATV. The factor "Attractiveness" of the UEQ was rated pleasant and likeable and as we know people tend to decrease the

distance to agents they like. These findings are supported by the results of the Godspeed Questionnaire. Participants felt secure and found the robot likeable. 


\section{Conclusion and Outlook}

In conclusion, we gained insights in how the social ATV is being perceived and could adjust the design and behaviour of it to optimize the collaboration with the human worker. The height influences the distance kept to the robot whereas the chosen velocities do not affect it. Small effects have been found between the two emotional representations. This is caused by the position of the display and the experience of the participant with robots. To communicate with its surrounding the display should always be at the highest position. Further, the facial features need to be stronger for experienced people.

The next steps in evaluating the interaction between the social robot and its user is to abstract design guidelines concerning the appearance of social robots in HRC. For this reason, further theoretical concepts regarding emotion regulation, such as emotional reactivity, should also be considered [4]. Furthermore, we consider to evaluate the multiple interaction modalities, like speech recognition and gesture control with mobile devices such as tablets or smartphones, and test them in usability tests. Concerning proxemics, the minimum distance in which the user is willing or feels comfortable to communicate with the ATV or the user notices the display of it in the first place, has to be identified. We will conduct this experiment in a real-world industrial case study. Since the robot's appearance may be compared to pets, we also think about including questions of preliminary experience about and ownership of pets in the questionnaire.

\section{References}

1. Optitrack motion capture system, http://optitrack.com/

2. Argyle, M., Dean, J.: Eye-contact, distance and affiliation. Sociometry 28(3), 289304 (1965), http://www.jstor .org/stable/2786027

3. Bartneck, C., Croft, E., Kulic, D.: Measurement instruments for the anthropomorphism, animacy, likeability, perceived intelligence, and perceived safety of robots. International Journal of Social Robotics 1(1), 71-81 (2009). https://doi.org/10.1007/s12369-008-0001-3

4. Becerra, R., Campitelli, G.: Emotional reactivity: Critical analysis and proposal of a new scale 3(6), 161-168 (2013). https://doi.org/10.5923/j.ijap.20130306.03

5. Boyle, E., Anderson, A., Newlands, A.: The effects of visibility on dialog and performance in a cooperative problem-solving task 37, 1-20 (01 1994)

6. Breazeal, C.: Affective interaction between humans and robots. In: Kelemen, J., Sosík, P. (eds.) Advances in Artificial Life. ECAL 2001. Lecture Notes in Computer Science. vol. 2159. Springer, Berlin, Heidelberg (2001)

7. Butler, J.T., Agah, A.: Psychological effects of behavior patterns of a mobile personal robot. Auton. Robots 10(2), 185-202 (Mar 2001). https://doi.org/10.1023/A:1008986004181, https://doi.org/10.1023/A : 1008986004181

8. Deutsches Institut für Normung: DIN EN ISO 10218:2012 Industrieroboter - Sicherheitsanforderungen (2012) 
9. Hall, E.: The hidden dimension. Doubleday Anchor Books, Doubleday (1966), https://books.google.de/books?id=u-wyAAAAMAAJ

10. Hiroi, Y., Ito, A.: Are bigger robots scary? -the relationship between robot size and psychological threat. In: 2008 IEEE/ASME International Conference on Advanced Intelligent Mechatronics. pp. 546-551 (July 2008). https://doi.org/10.1109/AIM.2008.4601719

11. Ikeura, R., Otsuka, H., Inooka, H.: Study on emotional evaluation of robot motions based on galvanic skin reflex. The Japanese journal of ergonomics 31(5), 355-358 (1995). https://doi.org/10.5100/jje.31.355

12. International Organization for Standardization: ISO 8373:2012-03 Robots and robotic devices - Vocabulary. (2012)

13. Jost, J., Kirks, T., Mättig, B., Sinsel, A., Trapp, T.U.: Der Mensch in der Industrie - Innovative Unterstützung durch Augmented Reality, pp. 153-174. Springer Berlin Heidelberg, Berlin, Heidelberg (2017)

14. Laugwitz, B., Held, T., Schrepp, M.: Construction and evaluation of a user experience questionnaire. In: Proceedings of the 4th Symposium of the Workgroup Human-Computer Interaction and Usability Engineering of the Austrian Computer Society on HCI and Usability for Education and Work. pp. 63-76. USAB '08, Springer-Verlag, Berlin, Heidelberg (2008)

15. Matthias, B., Ding, H., Miegel, V.: Die Zukunft der Mensch-Roboter Kollaboration in der industriellen Montage (10 2013)

16. Mutlu, B., Forlizzi, J., Hodgins, J.: A storytelling robot: Modeling and evaluation of human-like gaze behavior. In: 2006 6th IEEE-RAS International Conference on Humanoid Robots. pp. 518-523 (Dec 2006). https://doi.org/10.1109/ICHR.2006.321322

17. Nomura, T., Kanda, T., Suzuki, T., Kato, K.: Psychology in human-robot communication: An attempt through investigation of negative attitudes and anxiety toward robots. In: 2004 IEEE International Workshop on Robot and Human Interactive Interactive Communication. pp. 35-40. Kurashiki, Okayama Japan (Sep 2004)

18. Onnasch, L., Maier, X., Jürgensohn, T.: Mensch-Roboter-Interaktion - Eine Taxonomie für alle Anwendungsfälle., baua:Fokus, vol. 1 (2016)

19. Reeves, B., Nass, C.: The media equation: How people treat computers, television, and new media like real people and places (01 1996)

20. Sakai, T., Nakajima, H., Nishimura, D., Uematsu, H., Kitano, Y.: Autonomous mobile robot system for delivery in hospital. Technical Report of Matsushita Electric Works 53(2), 62-67 (2005)

21. Syrdal, D.S., Dautenhahn, K., Koay, K.L., Walters, M.L.: The negative attitudes towards robots scale and reactions to robot behaviour in a live human-robot interaction study (2009)

22. Weber, M.: Mensch-Roboter-Kollaboration (2001), https://www . arbeitswissenschaft.net/fileadmin/Downloads/Angebote_und_Produkte/ Zahlen_Daten_Fakten/ifaa_Zahlen_Daten_Fakten_MRK.pdf 